\title{
Berberine Suppresses Mice Depression Behaviors and Promotes Hippocampal Neurons Growth Through Regulating the miR-34b-5p/miR-470-5p/ BDNF Axis
}

This article was published in the following Dove Press journal:

Neuropsychiatric Disease and Treatment

Yuhua Zhan

Jiyang Han

Jing Xia

Xumei Wang

Department of Psychiatry, Shengjing Hospital of China Medical University,

Shenyang City, Liaoning Province,

People's Republic of China
Correspondence: Xumei Wang Department of Psychiatry, Shengjing Hospital of China Medical University, Shenyang City, Liaoning Province, People's Republic of China

$\mathrm{Tel} / \mathrm{Fax}+86$ 0249-66I566III2

Email xiaoyema2020@I63.com
Background: Berberine has been found to inhibit the progression of depression disorder, but its specific mechanism is still unclear. MicroRNA (miRNA) is considered to play an important role in the progression of depression. However, it is unclear whether Berberine is involved in the regulation of depression progression through miRNA.

Methods: The chronic unpredictable mild stress (CUMS) mice model was constructed. Mice depression behaviors were evaluated by sucrose preference test (SPT) and forced swim test (FST). Quantitative real-time PCR was employed to assess the expression of miR-34b$5 p, \operatorname{miR}-470-5 p$ and brain-derived neurotrophic factor (BDNF). The protein expression of BDNF was examined using Western blot analysis. In addition, the viability and apoptosis of hippocampal neurons were determined using cell counting kit 8 assay, flow cytometry and TUNEL assay. The interaction between BDNF and miR-34b-5p or miR-470-5p was verified by dual-luciferase reporter assay and RNA immunoprecipitation assay.

Results: Our data indicated that Berberine could inhibit CUMS mice depression behaviors and enhance hippocampal neurons growth by targeting miR-34b-5p and miR-470-5p. In addition, we found that BDNF was a target of miR-34b-5p and miR-470-5p. Overexpressed BDNF could reverse the regulation of miR-34b-5p and miR-470-5p on CUMS mice depression behaviors and hippocampal neurons growth. Furthermore, Berberine could promote BDNF expression to regulate CUMS mice depression behaviors and hippocampal neurons growth.

Conclusion: Berberine might inhibit the progression of depression disorder by regulating the miR-34b-5p/miR-470-5p/BDNF axis.

Keywords: depression disorder, Berberine, miR-34b-5p, miR-470-5p, BDNF

\section{Introduction}

Depression disorder is a chronic mood disorder disease characterized by low emotions, delayed thinking, and delayed speech and action. ${ }^{1,2}$ Depression disorder not only seriously the lives of patients, but also brings a heavy burden to the family and society. ${ }^{3,4}$ Chronic unpredictable mild stress (CUMS) is a widely used animal depression model at present, which is characterized by depressive symptoms such as loss of appetite, weight loss, loss of interest, decreased sensitivity, or anhedonia after animals have been subjected to different random stimuli for a long time., Compared with other animal models (eg chronic social defeat stress model) ${ }^{7-10}$ the 
CUMS model simulates the impact of various factors such as social life and environment on humans, and can more truly reflect the pathological process of major depression. $^{11,12}$

Berberine is an antibacterial alkaloid extracted from Berberis plants such as Coptis and Phellodendron, which is often used clinically to treat diarrhea. ${ }^{13,14}$ Modern pharmacological studies have confirmed that Berberine has important application prospects in cardiovascular, nervous system and mental diseases. ${ }^{15-17}$ Studies have reported that Berberine can inhibit the development of depression disorder, ${ }^{18,19}$ but the specific mechanism is not completely clear.

MicroRNA (miRNA) is a non-coding RNA with a length of about 22 nucleotides, and it is related to the progression of many diseases, including depression disorder. ${ }^{20,21}$ Previous studies have shown that the abnormal expression of let-7e, miR-146a and miR-155 is related to the severity of depression disorder. $^{22}$ MiR-34b-5p was found to be significantly upregulated in the peripheral blood leukocytes of patients with major depression disorder, ${ }^{23}$ and had markedly increased expression in mice under CUMS program. ${ }^{24}$ In addition, miR-470-5p had also been demonstrated to be elevated in CUMS-induced depression mice. ${ }^{25}$ Unfortunately, it is unclear whether miR$34 b-5 p$ and miR-470-5p can regulate depressive behavior.

Brain-derived neurotrophic factor (BDNF) is a protein synthesized in the brain and plays an important role in the survival, differentiation, growth and development of neurons. $^{26,27}$ Numerous studies have demonstrated that BDNF is a key sensor for antidepressant drugs and is of great significance for the treatment of depression disorder. $^{28,29}$ It has been reported that BDNF is downregulated in CUMS mice, which can participate in depression disorder development and hippocampal neurons injury. $^{30,31}$

In our research, we found that Berberine could regulate CUMS mice depressive behavior and hippocampal neurons growth, as well as regulate the expression of miR-34b-5p, miR-470-5p and BDNF. In addition, bioinformatics analysis found that both miR-34b-5p and miR-470-5p could target BDNF. Therefore, we hypothesized that Berberine might suppress progression of depression disorder by regulating the miR-34b-5p/miR-470-5p/BDNF axis.

\section{Materials and Methods}

\section{Animals}

Male C57BL/6J mice (8 weeks old) were obtained from Vital River (Beijing, China). All mice were kept in a 12 $\mathrm{h}$ light/dark cycle space and given free access to food and water. All animal protocols were approved by Ethics Committee of Shengjing Hospital of China Medical University and performed according to the Guide for the Care and Use of Laboratory Animals (Eighth Edition, National Research Council).

\section{CUMS Program}

During the modeling process, the mice were kept in single cages. The CUMS program was carried out in accordance with previous research methods with minor modifications. ${ }^{32}$ The following various pressure stimuli were given to mice within 5 weeks. Including water forbidden for $24 \mathrm{~h}$, fasting for $24 \mathrm{~h}$, peculiar smell, wet cage for $24 \mathrm{~h}$, restraint $(3 \mathrm{~h})$, pinching $(2 \mathrm{~min}, 2 \mathrm{~cm}$ from the tip of the tail), and noise. Mice received 1 stressor randomly every day for 5 weeks. The mice in the control group did not receive any stimulation. Then, all mice were performed the behavioral tests.

\section{Sucrose Preference Test (SPT)}

The mice were raised individually before the experiment. During the training period, 2 bottles of $1 \%(\mathrm{w} /$ v) sucrose solution were placed in the squirrel cage for $24 \mathrm{~h}$. After that, one of the bottles was changed to pure water, and after $12 \mathrm{~h}$ of adaptation, the position of sucrose water and pure water were changed to continue to adapt for $12 \mathrm{~h}$. After the mice were deprived of water and food for $24 \mathrm{~h}, 1$ bottle of water and 1 bottle of $1 \%$ $(\mathrm{w} / \mathrm{v})$ sucrose solution were placed in cages with free access. $24 \mathrm{~h}$ later, the remaining amounts of sucrose and water were determined. The sucrose preference (\%) was determined using: sucrose preference $(\%)=$ sucrose consumption/(water consumption + sucrose consumption) $\times 100 \%$.

\section{Forced Swim Test (FST)}

The mice were putted in a cylindrical container (water depth of about $30 \mathrm{~cm}$ ) filled with clean water, and was force to swim. A camera system was used to record the swimming time and immobility time of mice within 5 min. Swimming time referred to the time when the mice upper body or head on the surface of the water and swimming continuously. The immobility time referred to the time when the mice had its head out of the water, floating motionless, or occasionally swimming forelegs to keep the body in balance. 


\section{Adenovirus Injection and Berberine Treatment}

MiR-34b-5p and miR-470-5p overexpressing adenovirus vectors (Ad-miR-34b-5p and Ad-miR-470-5p) and their controls (Ad-miR-NC), BDNF overexpressing adenovirus vector or short guide RNA (sgRNA) adenovirus vector (Ad-BDNF and sg-BDNF) and their controls (Ad-NC and sg-NC) were constructed from Hanbio (Shanghai, China). CUMS mice were anesthetized by intraperitoneal injection of $10 \%$ chloral hydrate $(4 \mathrm{~mL} / \mathrm{kg}$, Sigma-Aldrich, St. Louis, MO, USA) and fixed on a stereotactic instrument. A $0.5 \mathrm{~mm}$ diameter drill was used to open the skull on both sides of the mice. Subsequently, the adenovirus vectors $\left(10^{11} \mathrm{pfu} / \mathrm{mL}, 1 \mu \mathrm{L}\right)$ were slowly injected into the hippocampus using a glass microtube fixed on the stereotaxic instrument. After the operation, the mice were taken out of the stereotaxic instrument and placed in a warm cage to recover. On the third day after the operation, $20 \mathrm{mg} / \mathrm{kg}$ /day Berberine (dissolved in normal saline; Guangrui Bio, Shanghai, China) was administered by gavage. 2 weeks after the surgery, the mice were subjected to behavioral tests. In addition, the mice were euthanized and their hippocampus tissues were taken to detect the expression of miRNA and BDNF.

\section{Hippocampal Neurons Transfection and Berberine Treatment}

Mice hippocampal neurons were obtained from Procell Life Science \& Technology (Wuhan, China) and cultured in complete culture medium for mice hippocampal neurons (CM-M107, Procell Life Science \& Technology). MiR-34b-5p and miR-470-5p mimics (miR-34b-5p and miR-470-5p) and inhibitors (anti-miR-34b-5p and antimiR-470-5p), BDNF pcDNA overexpression vector and small interfering RNA (siRNA) (BDNF and si-BDNF) and their negative controls (miR-NC, anti-miR-NC, pcDNA and si-NC) were obtained from Genepharma (Shanghai, China). Lipofectamine 3000 reagent (Invitrogen, Carlsbad, CA, USA) was used for cell transfection. After transfection for $24 \mathrm{~h}$, hippocampal neurons were treated with $10^{-5} \mathrm{~mol} / \mathrm{L}$ Berberine for $24 \mathrm{~h}$.

\section{Quantitative Real-Time PCR (qRT-PCR)}

After extracted RNA from the hippocampus tissues and hippocampal neurons using TRIzol reagent (Invitrogen), the RNA was reverse-transcribed into cDNA using the HiScript $^{\circledR}$ II One Step RT-PCR Kit (Vazyme, Nanjing,
China). QRT-PCR was performed using SYBR Green (Solarbio, Beijing, China) on PCR system. U6 or GAPDH was utilized as the internal control for miRNA and BNDF, respectively. Relative expression was determined using the $2^{-\Delta \Delta \mathrm{CT}}$ method. The primers used in this research were as below: miR-34b-5p, 5'CGAGGCAGTGTAATTAGCTGATTGT-3'; miR-470-5p, F, 5'-GTGCGAACCAGTACCTTTCTG-3', R 5'GTGCAGGGTCCGAGGT-3'; U6， F 5'-GCTTCGGCA GCACATATACTAAAAT-3', R 5'-CGCTTCACGAATTT GCGTGTCAT-3'; BDNF, F 5'-AAGGACGCGGACT TGTACAC-3', R 5'-CGCTAATACTGTCACACACGC -3'; GAPDH, F 5'-CGTCCCGTAGACAAAATGGT-3', R 5'-TTGATGGCAACAATCTCCAC-3'.

\section{Western Blot (WB) Analysis}

Hippocampus tissues and hippocampal neurons were treated with ice-cold RIPA lysis buffer (Beyotime, Shanghai, China) to extract protein. After determining the protein concentrations using BCA Assay Kit (Beyotime), protein samples were separated in SDS-PAGE gels and then transferred onto PVDF membranes (Invitrogen). Subsequently, the membrane was incubated with skim milk, primary antibody against BDNF (1:2000, Boster, Beijing, China) or GAPDH (1:2000, Boster), and secondary antibody (Goat anti-rabbit, 1:10,000, Boster) in turn. The signal intensity was determined by Super ECL Detection Reagent (Yeasen, Shanghai, China).

\section{Cell Counting Kit 8 (CCK8) Assay}

Hippocampal neurons were seeded in 96-well plates. After incubating for $48 \mathrm{~h}$, the cells were added with CCK8 solution (10 $\mu \mathrm{L}$, Dojindo, Kumamoto, Japan) for $4 \mathrm{~h}$, the absorbance was analyzed at $450 \mathrm{~nm}$ using a microplate reader to evaluate cell viability.

\section{Flow Cytometry}

The apoptosis of hippocampal neurons was detected by Annexin V-FITC Apoptosis Detection Kit (Dojindo). Hippocampal neurons were collected and then suspended with $1 \times$ Binging Buffer to obtain the cell suspensions. Subsequently, the cell suspensions were stained with Annexin V-FITC and Propidium iodide for $30 \mathrm{~min}$. The apoptosis rate of cells was examined by a flow cytometry.

\section{TUNEL Assay}

TUNEL assay reagent was obtained from Merck KGaA (Darmstadt, Germany). According to the kit instructions, 
TUNEL positive cells in hippocampal neurons were analyzed under microscopy.

\section{Dual-Luciferase Reporter Assay}

The sequences of BDNF 3 'UTR containing the binding sites or mutant sites with miR-34b-5p or miR-470-5p were amplified and fused into the psiCHECK2 luciferase vector (Promega, Madison, WI, USA) to generate the WT/MUTBDNF 3'UTR-1 or WT/MUT-BDNF 3'UTR-2 vectors, respectively. Then, $20 \mathrm{ng}$ vectors and $50 \mathrm{nM}$ of miRNAs mimic or miR-NC were co-transfected into HEK293T cells (24-well plates, Procell Life Science \& Technology). Relative luciferase activities (Firefly/ Renilla) were assessed after $48 \mathrm{~h}$ using Dual-Glo ${ }^{\circledR}$ Luciferase Assay System (Promega).

\section{RNA Immunoprecipitation (RIP) Assay}

RIP Kit (Millipore, Billerica, MA, USA) was used to perform this assay. Hippocampal neurons were lysed to collect cell suspensions. After suspending with RIP lysis buffer, cell suspensions were incubated with magnetic beads pre-coated with Ago2 antibody (1:20, Boster) or IgG antibody (1:50, Boster) to get the immunoprecipitation complex. The relative RNA enrichments of miR-34b$5 p$, miR-470-5p and BDNF were measured by qRT-PCR.

\section{Statistical Analysis}

All experiments were performed in triplicate and all data were shown as mean \pm standard deviation. GraphPad Prism 7 software (GraphPad, La Jolla, CA, USA) was employed to perform statistical analysis. The statistical significance was analyzed using Student's $t$-test or oneway analysis of variance followed by a Tukey post-hoc test. The correlation between BDNF and miR-34b-5p or miR-470-5p was analyzed using Pearson correlation analysis. $P<0.05$ was considered statistically significant.

\section{Results}

\section{Berberine Ameliorated Depressive-Like} Behavior in CUMS Mice and Regulated miR-34b-5p, miR-470-5p and BDNF Expression

To confirm the role of Berberine in depression disorder, C57BL/6J mice were received a 5-week CUMS program. After exposed to CUMS, the sucrose preference and swimming time of mice were significantly reduced, while the treatment of Berberine could increase the sucrose preference and swimming time of CUMS mice (Figure $1 \mathrm{~A}$ and $\mathrm{B})$. Also, the immobility time of the mice exposed to CUMS was markedly enhanced, while Berberine could effectively decrease the immobility time of CUMS mice (Figure 1C). In the hippocampus of CUMS mice, we found that miR-34b-5p and miR-470-5p were remarkably upregulated compared to the control mice, and this effect could be significantly inhibited by Berberine (Figure 1D and E). In addition, the mRNA and protein expression levels of BDNF were markedly downregulated in the hippocampus of CUMS mice (Figure $1 \mathrm{~F}$ and $\mathrm{G}$ ). Surprisingly, correlation analysis showed that BDNF mRNA expression was negatively correlated with miR$34 b-5 p$ and miR-470-5p expression in the hippocampus of CUMS mice (Figure $1 \mathrm{H}$ and I). These data showed that miR-34b-5p, miR-470-5p and BDNF might have important roles in the regulation of in Berberine regulating depressive-like behavior.

\section{Berberine Regulated CUMS Mice Depressive Behavior and Hippocampal Neurons Growth by Targeting miR-34b- $5 p$}

To explore the role of miR-34b-5p in depression disorder, miR-NC or miR-34b-5p overexpressing adenovirus were injected into the hippocampus of CUMS mice, followed by treating with Berberine. By measuring miR-34b-5p expression, we found that the miR-34b-5p expression was markedly enhanced in the hippocampus of CUMS mice injected with miR-34b-5p overexpressing adenovirus, while this effect could be inhibited by Berberine treatment (Figure 2A). Function experiments showed that overexpressed miR-34b-5p could obviously decrease the sucrose preference and swimming time, and significantly increase the immobility time of CUMS mice, while the treatment of Berberine could effectively reverse the promotion effect of miR-34b-5p on the depressive-like behavior of CUMS mice (Figure 2BD). To further confirm the function of miR-34b-5p, miR34b-5p mimic was transfected into hippocampal neurons, and then treated with Berberine. The detection results of cell viability and apoptosis suggested that miR-34b-5p overexpression notably inhibited cell viability and significantly promoted cell apoptosis, while these effects could be reversed by Berberine (Figure 2E and F). Furthermore, miR-34b-5p also markedly enhanced the TUNEL positive cells in hippocampal neurons, and Berberine treatment also could abolish this effect (Figure 2G). All data confirmed that 

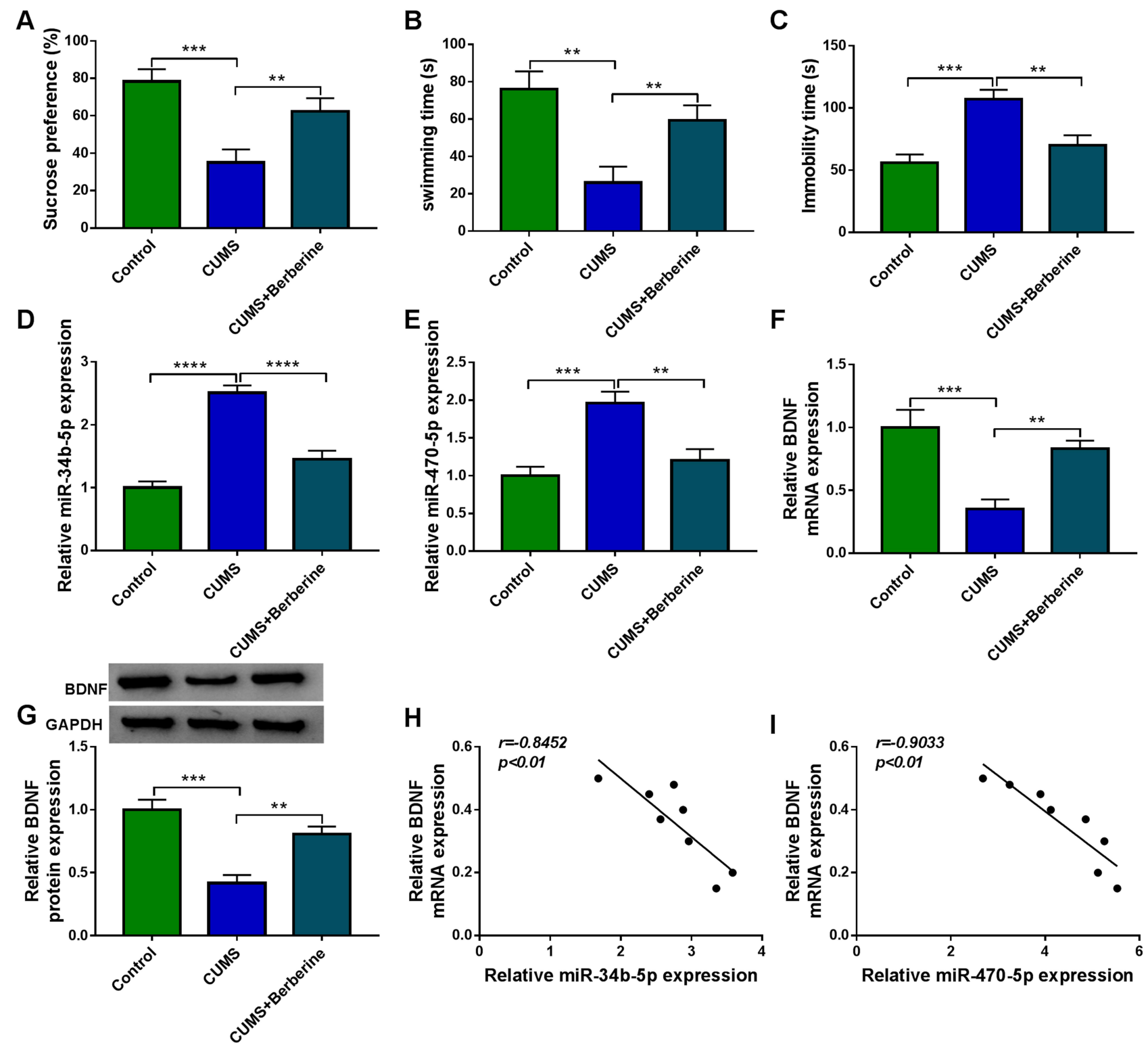

Figure I Berberine ameliorated depressive-like behavior in CUMS mice. C57BL/6 J mice were received a 5-week CUMS program with or without Berberine treatment. Non-treated mice were used as control. SPT (A) and FST (B and $\mathbf{C}$ ) were used to evaluate the sucrose preference, swimming time and immobility time of mice. (D and E) MiR-34b-5p and miR-470-5p expression in the hippocampus of CUMS mice and control mice was measured using qRT-PCR. (F and G) QRT-PCR and WB analysis were used to assess BDNF mRNA and protein expression in the hippocampus of CUMS mice and control mice. (H and I) Pearson correlation analysis was used to assess the correlation between BDNF mRNA expression and miR-34b-5p or miR-470-5p expression in the hippocampus of CUMS mice. $* * P<0.01$, $* * * P<0.00 \mathrm{I}$, $* * * * P<0.000 \mathrm{I}$.

Berberine inhibited miR-34b-5p expression to regulate depressive behavior and hippocampal neurons growth.

\section{Berberine Regulated miR-470-5p Expression to Mediate CUMS Mice Depressive Behavior and Hippocampal Neurons Growth}

Similarly, we evaluated the role of miR-470-5p in depression disorder by injecting the miR-470-5p overexpressing adenovirus into C57BL/6J mice. After injecting, miR-470-5p expression was indeed significantly increased the hippocampus of CUMS mice, and Berberine could effectively inhibit miR-470-5p expression (Figure 3A). Further studies showed that Berberine could reverse the inhibitory effect of miR-470$5 \mathrm{p}$ overexpression on the sucrose preference and swimming time, and the promoting effect on the immobility time in CUMS mice (Figure 3B-D). In hippocampal neurons transfected with miR-470-5p mimic, we discovered that miR-470$5 \mathrm{p}$ also repressed the viability and increased the apoptosis and TUNEL positive cells. However, the inhibition effect of miR470-5p on hippocampal neurons growth also could be reversed 


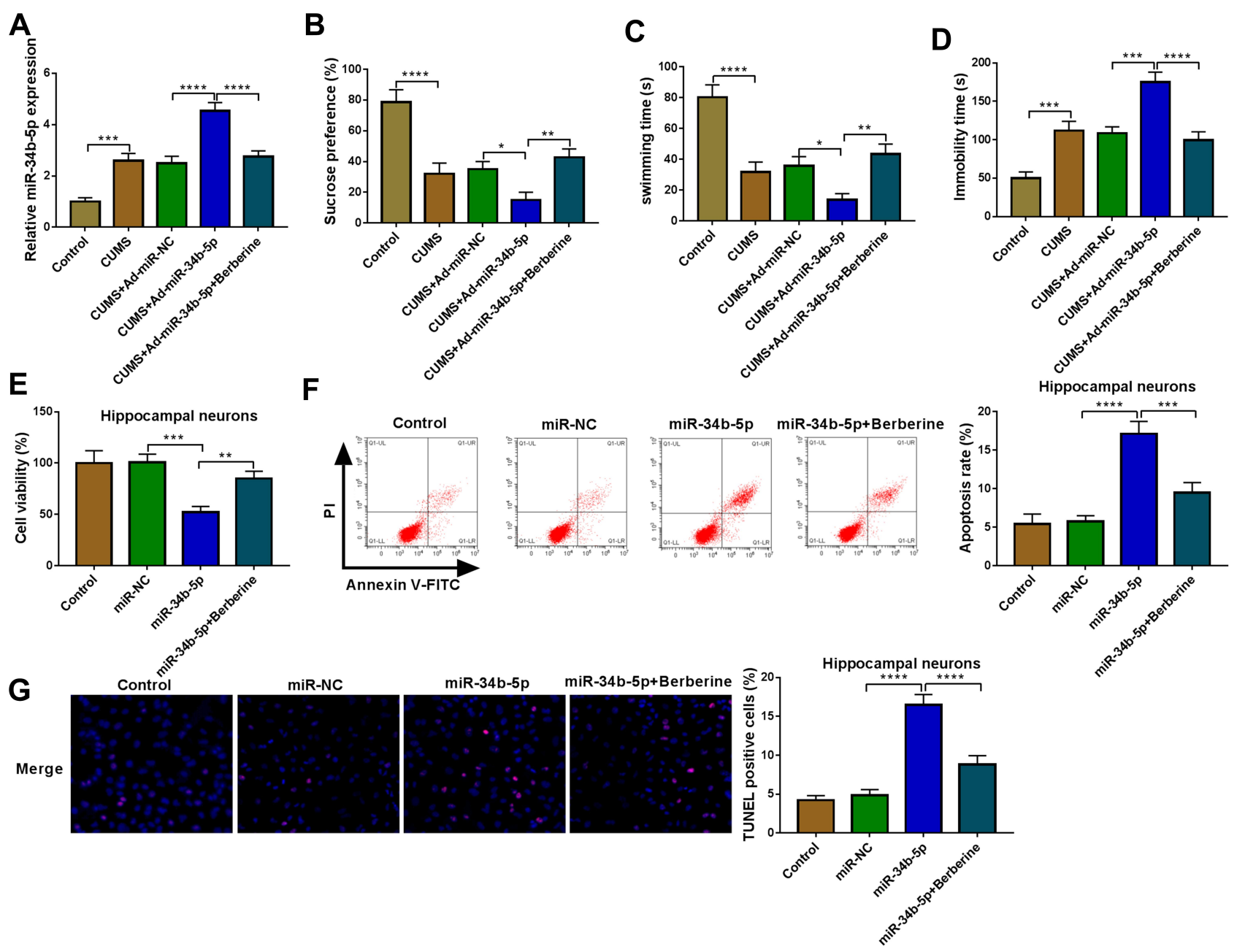

Figure 2 Berberine regulated CUMS mice depressive behavior and hippocampal neurons growth by targeting miR-34b-5p. (A-D) CUMS mice were divided into 5 groups, including control, CUMS, CUMS + Ad-miR-NC, CUMS + Ad-miR-34b-5p, CUMS + Ad-miR-34b-5p + Berberine. (A) QRT-PCR was used to test miR-34b-5p expression. The sucrose preference, swimming time and immobility time of mice were determined using SPT (B) and FST (C and D). (E-G) Hippocampal neurons were transfected with miR-34b-5p mimic or miR-NC, or treated with Berberine. Non-transfected and non-treated cells were used as control. (E) CCK8 assay was used to detect cell viability. The apoptosis rate and TUNEL positive cells were determined using flow cytometry $(\mathbf{F})$ and TUNEL assay $(\mathbf{G})$ to assess cell apoptosis. $* P<0.05$, $* * P<0.0$ I, $* * * P<0.00 \mathrm{I}$, $* * * * P<0.0001$.

by Berberine (Figure 3E-G). Therefore, our data revealed that Berberine also could hinder miR-470-5p expression to regulate depressive behavior and hippocampal neurons growth.

\section{BDNF Was a Target of miR-34b-5p and miR-470-5p}

Using the starBase v2.0 software, we found that BDNF had complementary binding sites with miR-34b-5p and miR-470$5 p$ (Figure 4A). Dual-luciferase reporter assay results determined that miR-34b-5p and miR-470-5p overexpression could reduce the luciferase activity of WT-BDNF-3'UTR-1 vector and WT-BDNF-3'UTR-2 vector, respectively, but had no effect on the luciferase activity of their corresponding MUT vectors (Figure 4B). Furthermore, the results of RIP assay suggested that the levels of miR-34b-5p and BDNF, as well as miR-470-5p and BDNF were significantly enriched in Ago2 (Figure 4C). In order to determine the regulation of miR-34b-5p and miR-470-5p on BDNF expression, hippocampal neurons were transfected with the mimics or inhibitors of miR-34b-5p and miR-470-5p. The significant increase or decrease of miR-34b-5p and miR-470-5p expression indicated that the transfections of miR-34b-5p and miR-470-5p mimics or inhibitors were effective (Figure 4D and E). Through measuring BDNF protein expression, we discovered that the BDNF protein expression could be inhibited by miR-34b-5p and miR-470-5p overexpression, while promoted by miR-34b-5p and miR-470-5p inhibition (Figure $4 \mathrm{~F}$ and $\mathrm{G})$. These results demonstrated that BDNF could be targeted by miR-34b-5p and miR-470-5p. 

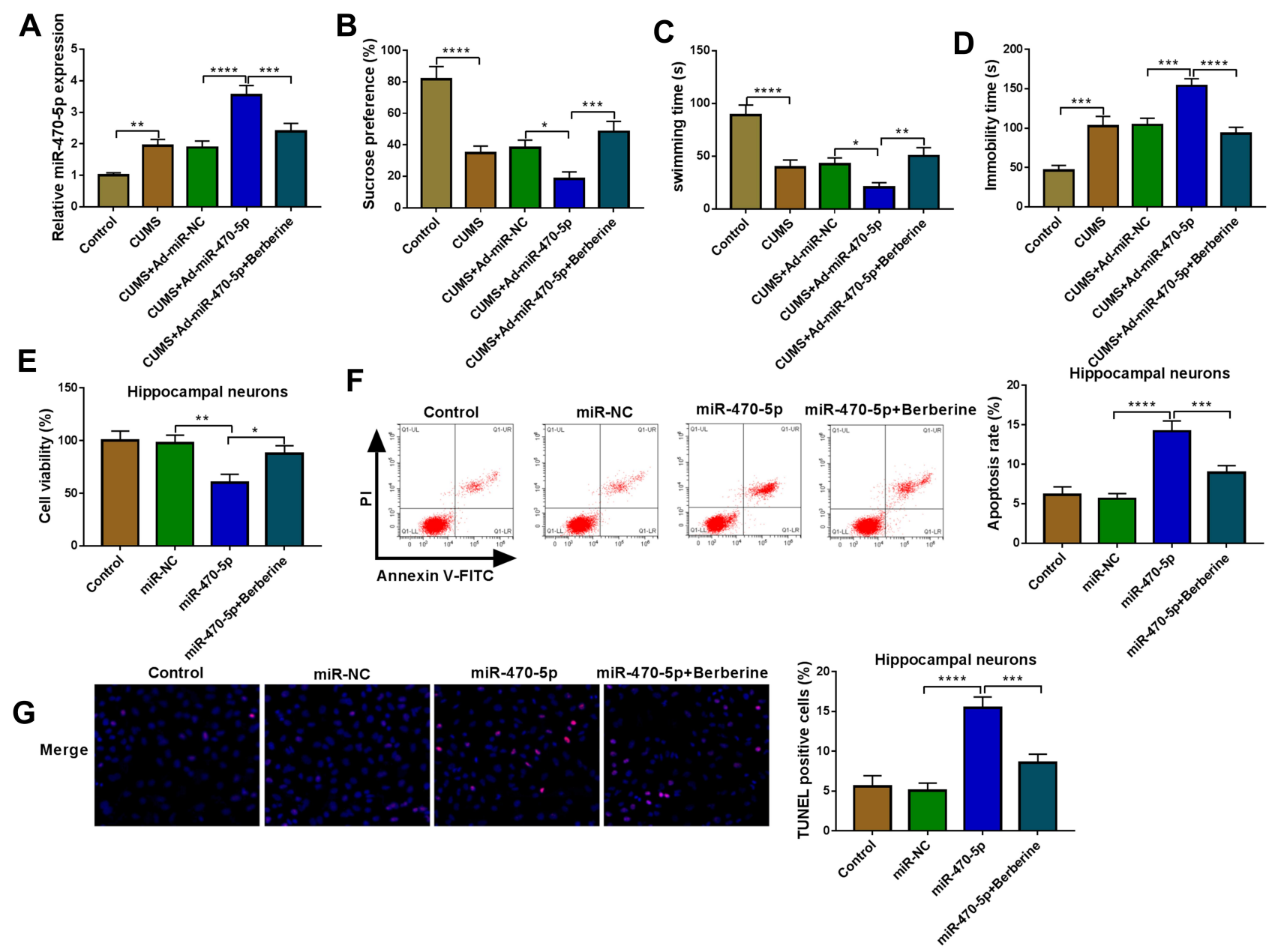

Figure 3 Berberine regulated miR-470-5p expression to mediate CUMS mice depressive behavior and hippocampal neurons growth. (A-D) C57BL/6) mice were divided into 5 groups, including control, CUMS, CUMS + Ad-miR-NC, CUMS + Ad-miR-470-5p, CUMS + Ad-miR-470-5p + Berberine. (A) QRT-PCR was used to test miR-470-5p expression. SPT (B) and FST (C and D) were performed to detect the sucrose preference, swimming time and immobility time of mice. (E-G) Hippocampal neurons were transfected with miR-470-5p mimic or miR-NC, or treated with Berberine. Non-transfected and non-treated cells were used as control. (E) CCK8 assay was utilized for measuring cell viability. Flow cytometry $(\mathbf{F})$ and TUNEL assay $(\mathbf{G})$ were used to assess the apoptosis rate and TUNEL positive cells to evaluate cell apoptosis. $* P<0.05$, $* * P<$ 0.01 , $* * * p<0.001$, $* * * * p<0.0001$.

\section{MiR-34b-5p and miR-470-5p Regulated CUMS Mice Depressive Behavior and Hippocampal Neurons Growth by Targeting BDNF}

To evaluate whether miR-34b-5p regulated CUMS mice depressive behavior and hippocampal neurons growth by targeting BDNF, we co-injected with miR-34b-5p overexpressing adenovirus and BDNF overexpressing adenovirus into mice. Overexpressed BDNF could effectively restore the inhibitory effect of miR-34b-5p mimic on BDNF expression in the hippocampus of CUMS mice (Figure 5A). By examining the depressive behavior in CUMS mice, we found that overexpression of BDNF significantly reversed the decreasing effect of miR-34b-
$5 \mathrm{p}$ on sucrose preference and swimming time, as well as the increasing effect on immobility time in CUMS mice (Figure 5B-D). Also, overexpressed BDNF abolished the inhibitory of miR-34b-5p on the viability, and the promotion effect on the apoptosis and TUNEL positive cells of hippocampal neurons (Figure 5E-H). In addition, we also co-injected with miR-470-5p overexpressing adenovirus and BDNF overexpressing adenovirus into mice. The increased BDNF protein expression in CUMS mice co-injected with miR-470-5p overexpressing adenovirus and BDNF overexpression vector confirmed the injection was successful (Figure 6A). Similarly, we also discovered that overexpressed BDNF could reverse the inhibition effect of miR-470-5p overexpression on sucrose preference and swimming time, and the enhancing effect 


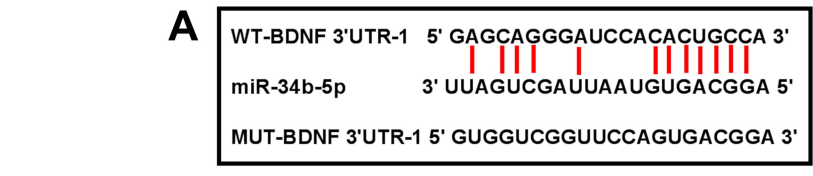

B

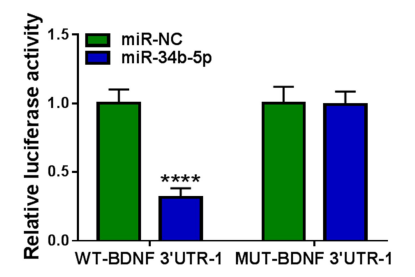

D

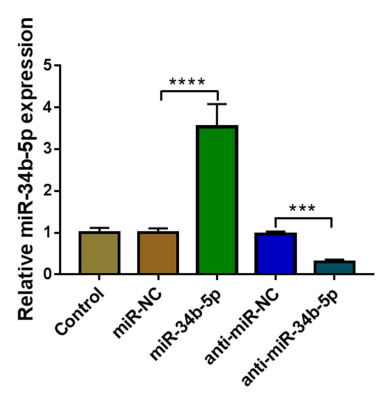

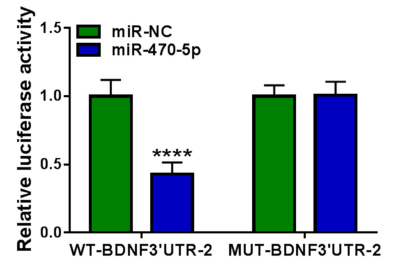

E

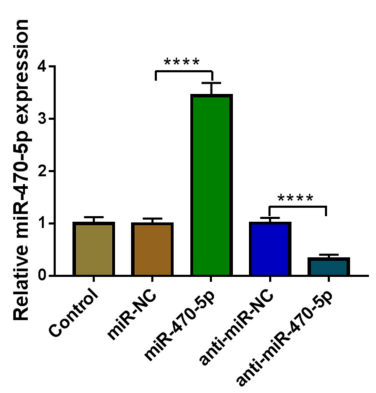

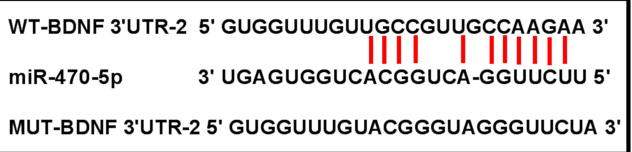

C
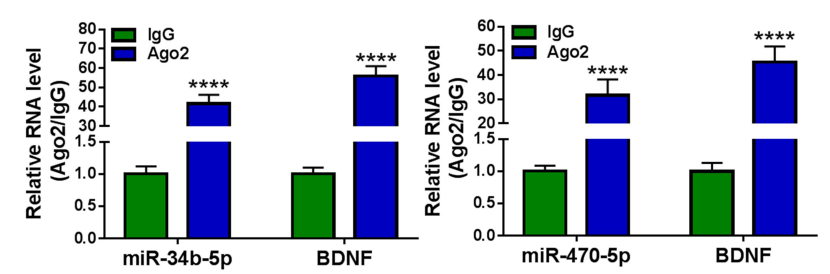

$\mathrm{F}_{\mathrm{BDNF}}=---$
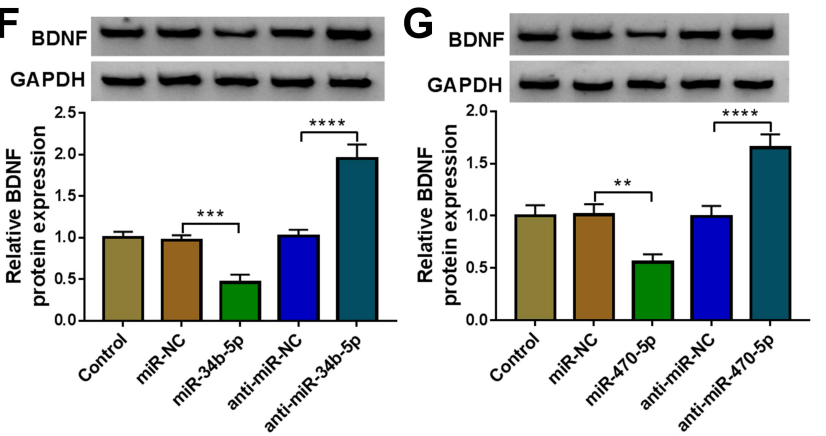

Figure 4 MiR-34b-5p and miR-470-5p directly interacted with BDNF. (A) The sequences of WT/MUT-BDNF 3'UTR-I and WT/MUT-BDNF 3'UTR-2 were shown. Dualluciferase reporter assay (B) and RIP assay (C) were used to evaluate the interaction between BDNF and miR-34b-5p or miR-470-5p. (D) The transfection efficiencies of miR-34b-5p mimic and inhibitor were evaluated by measuring miR-34b-5p expression in hippocampal neurons. (E) MiR-470-5p expression was determined using qRT-PCR to assess the transfection efficiencies of miR-470-5p mimic or inhibitor in hippocampal neurons. (F and $\mathbf{G})$ BDNF protein expression was tested by WB analysis in hippocampal neurons transfected with miR-34b-5p and miR-470-5p mimics or inhibitors. $* * p<0.01$, $* * * p<0.00 \mathrm{I}$, $* * * * P<0.000$ I.

on immobility time in CUMS mice (Figure 6B-D). Also, the suppressive effect of miR-470-5p on cell viability and the accelerating effect on apoptosis and TUNEL positive cells in hippocampal neurons also could be abolished by the addition of BDNF overexpression vector (Figure $6 \mathrm{E}-\mathrm{H}$ ). The above results confirmed that miR-34b-5p and miR-470-5p targeted BDNF to regulate depressive behavior and hippocampal neurons growth.

\section{Berberine Mediated BDNF Expression to Regulate CUMS Mice Depressive Behavior and Hippocampal Neurons Growth}

To further evaluate the role of BDNF in depression disorder, the sgRNA adenovirus vector of BDNF was injected into CUMS mice, followed by treating with Berberine. The detection of BDNF expression showed that BDNF silencing could markedly inhibit BDNF expression in the hippocampus of CUMS mice, while Berberine treatment could reverse this effect (Figure 7A). Further analysis revealed that knockdown of BDNF could reduce the sucrose preference, swimming time, and increase the immobility time in CUMS mice, while these effects also could be abolished by Berberine (Figure 7B-D). In hippocampal neurons transfected with si-BDNF, cell viability was markedly reduced, and cell apoptosis and TUNEL positive cells were significantly enhanced. However, the addition of Berberine also could reverse the suppressive effect of BDNF knockdown on hippocampal neurons growth (Figure 7E-G). Hence, we concluded that BDNF was involved in the regulation of Berberine on depression disorder and hippocampal neurons growth.

\section{Berberine Regulated BDNF Expression by Targeting miR-34b-5p and miR-470-5p in Hippocampal Neurons}

To further confirm whether Berberine regulated BDNF expression via miR-34b-5p and miR-470-5p, hippocampal neurons were transfected with miR-34b-5p or miR-470-5p mimics, followed by treating with Berberine. As shown in Figure $8 \mathrm{~A}$ and $\mathrm{B}$, we found that the inhibition effects of miR-34b-5p and miR-470-5p overexpression on the mRNA and protein expression of BDNF could be reversed by Berberine. Therefore, our data revealed that Berberine mediated CUMS mice depressive behavior and 


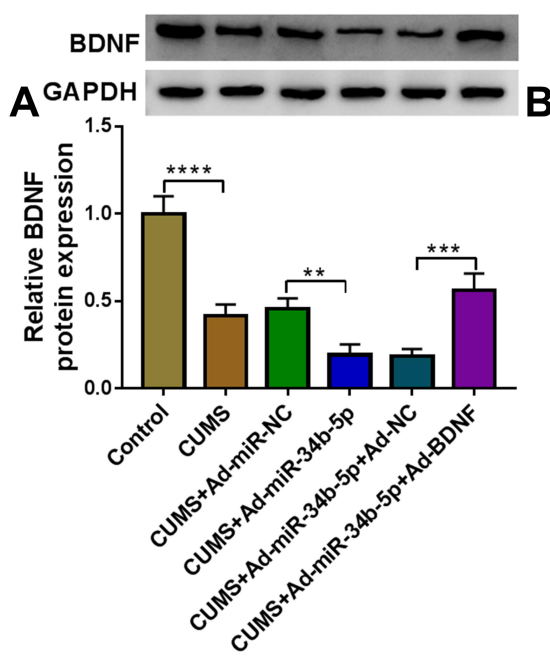

D

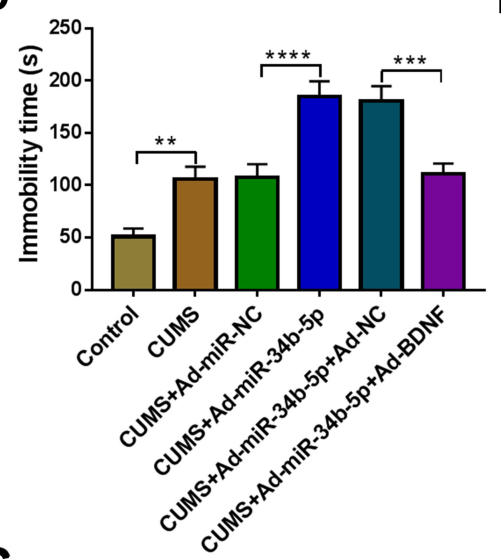

G

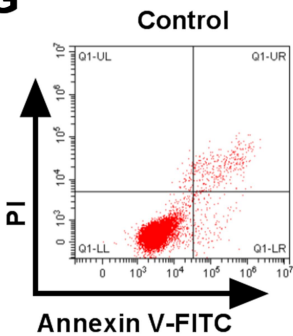

H

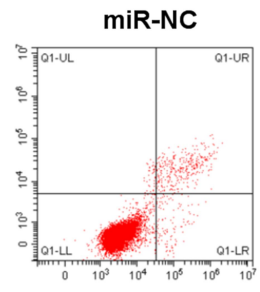

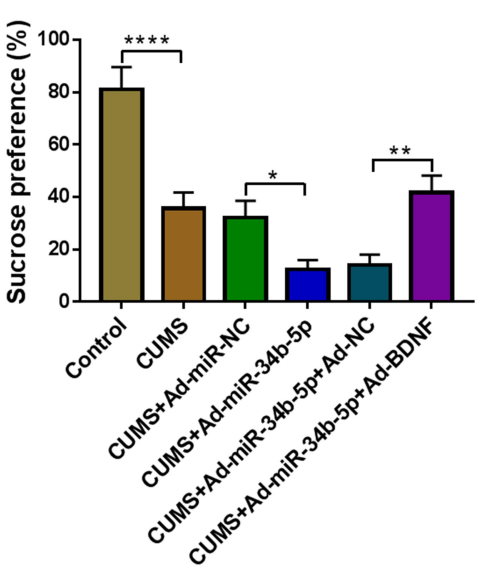

E

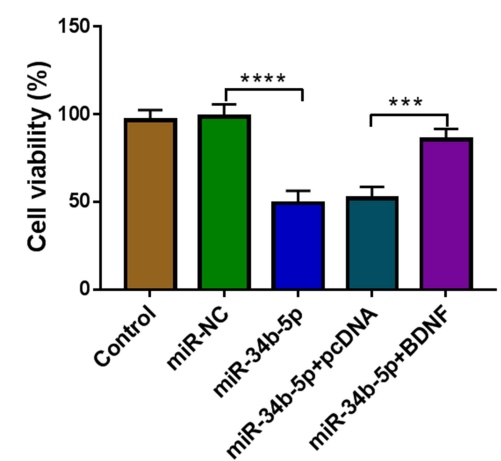

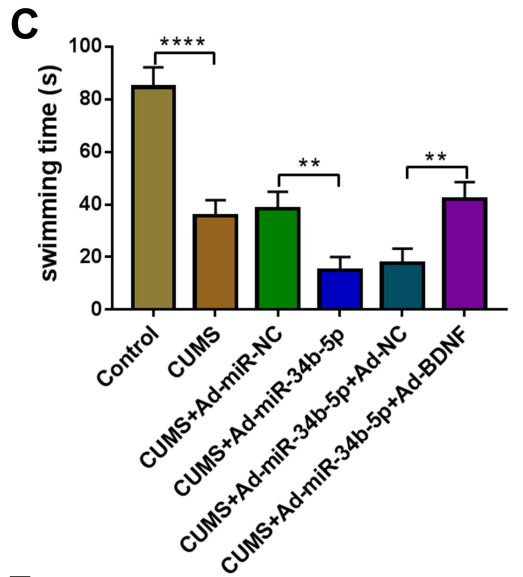

$\mathbf{F}$

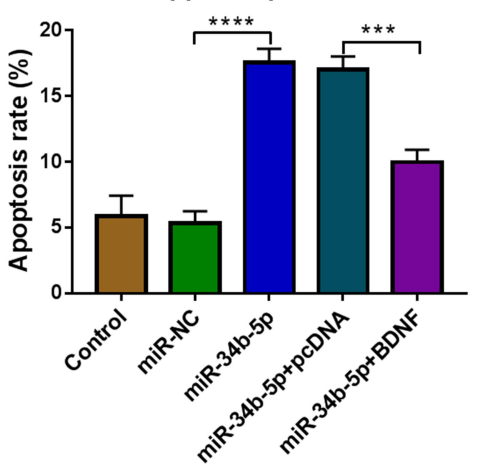

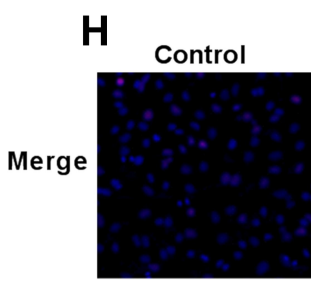
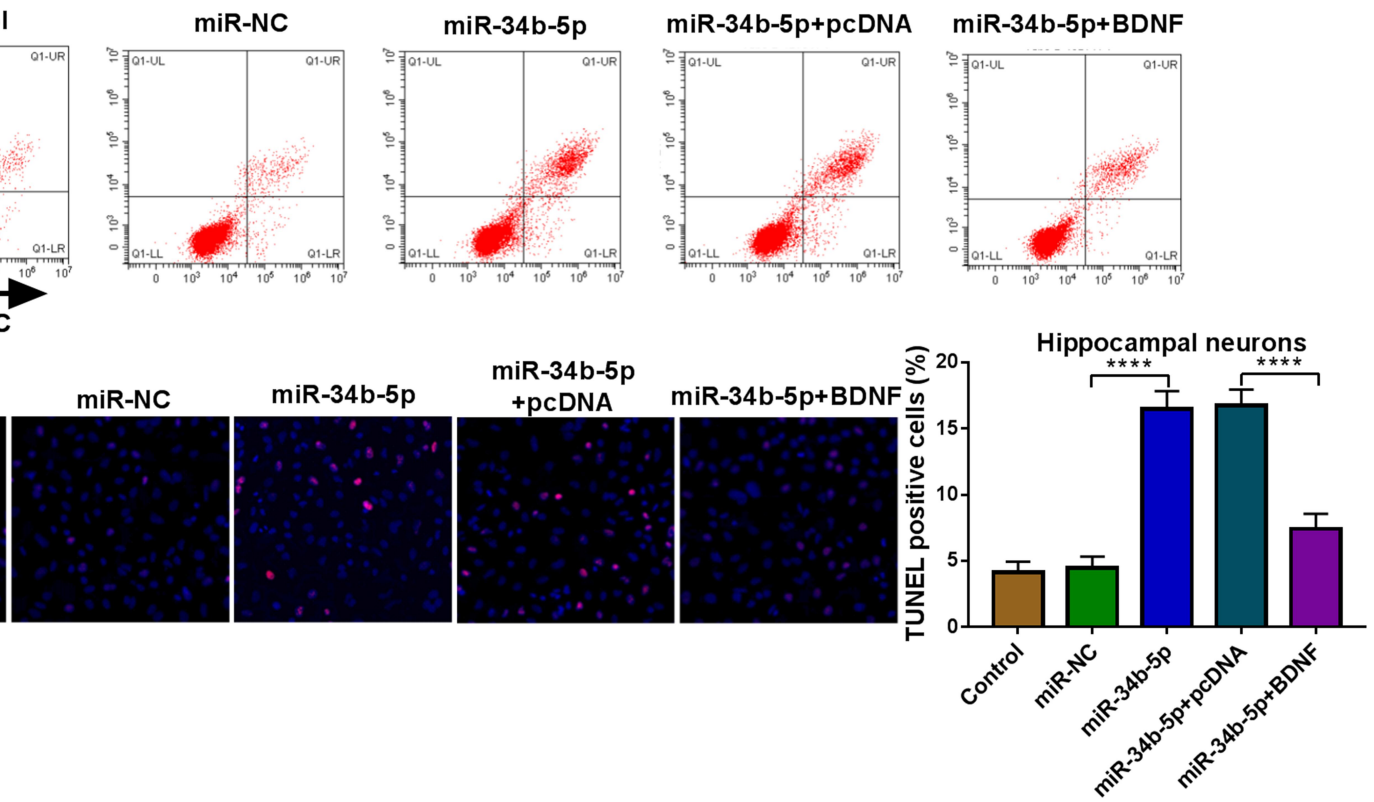

Figure 5 MiR-34b-5p and BDNF regulated CUMS mice depressive behavior and hippocampal neurons growth. (A-D) C57BL/6] mice were performed CUMS program, and then injected with Ad-miR-NC, Ad-miR-34b-5p, Ad-miR-34b-5p + Ad-NC or Ad-miR-34b-5p + Ad-BDNF. Non-treated mice were used as control. (A) WB analysis was used to test BDNF expression. SPT (B) and FST (C and D) were used to measure the sucrose preference, swimming time and immobility time of mice. (E-H) Hippocampal neurons were transfected with miR-NC, miR-34b-5p, miR-34b-5p + pcDNA or miR-34b-5p + BDNF. Non-transfected and non-treated cells were used as control. (E) Cell viability was measured using CCK8 assay. Flow cytometry $(\mathbf{F}$ and $\mathbf{G})$ and TUNEL assay $(\mathbf{H})$ were performed to detect cell apoptosis rate and TUNEL positive cells to assess cell apoptosis. $* P<0.05$, $* * P<0.01$, $* * * P<0.001$, $* * * * P<0.0001$. 


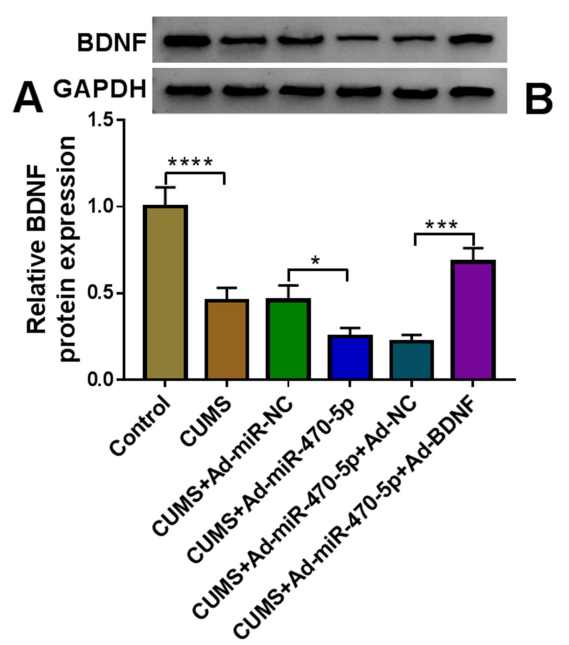

D

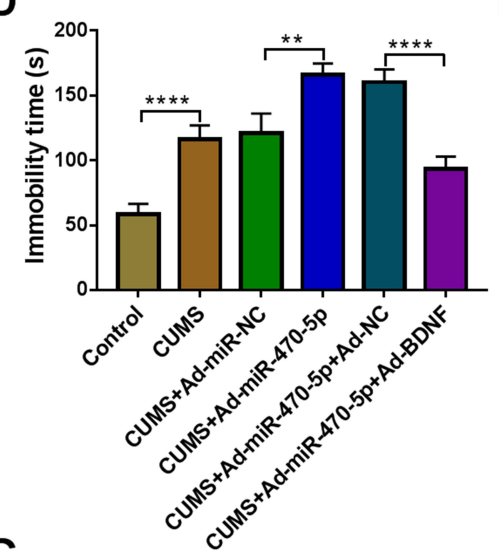

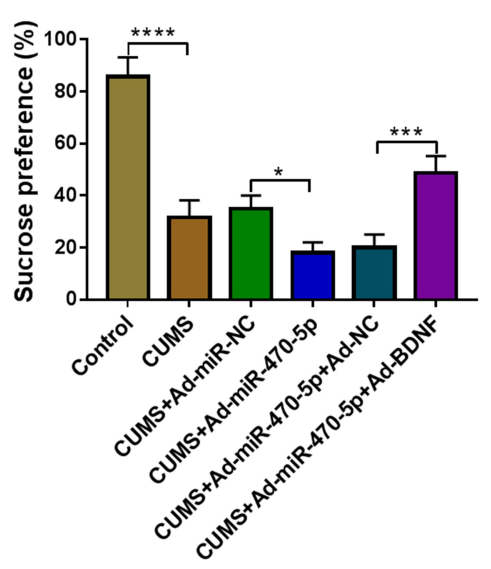

E

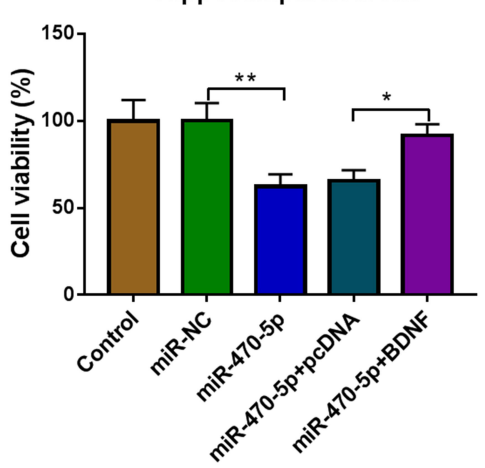

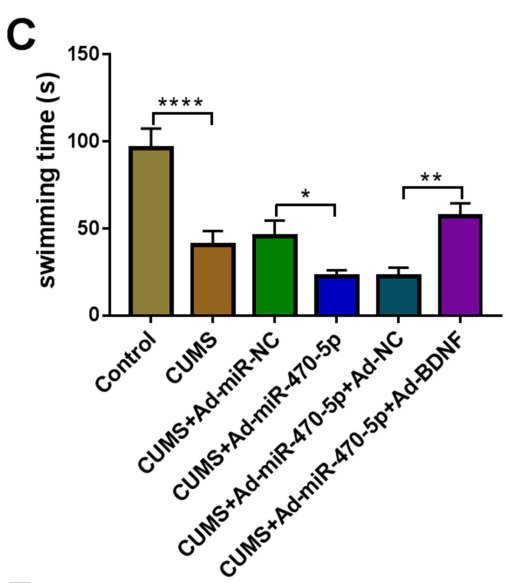

F

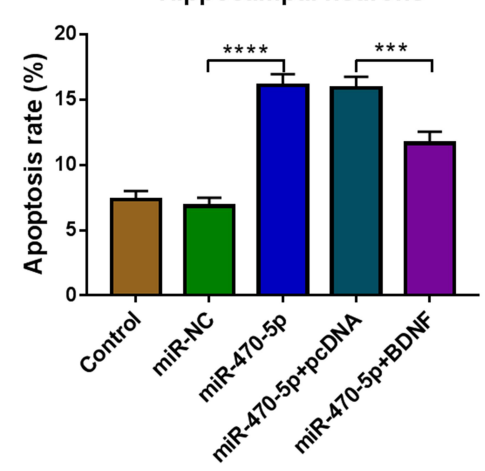

G
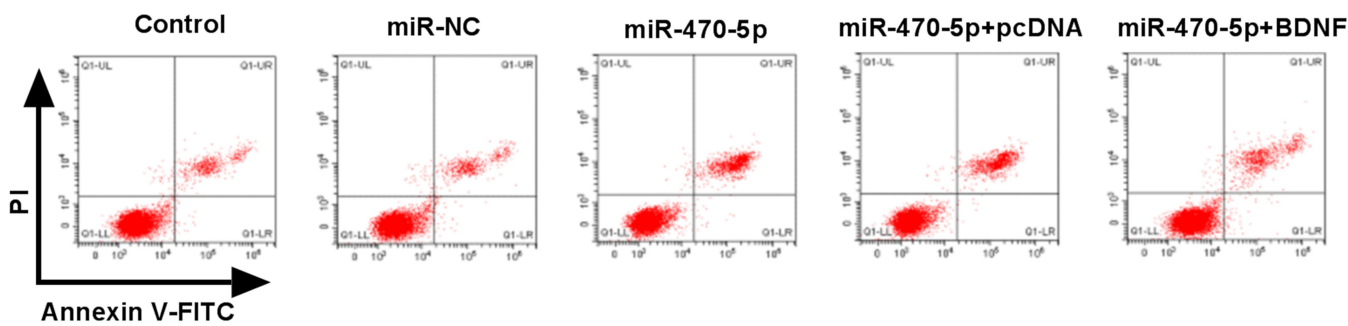

H

Control
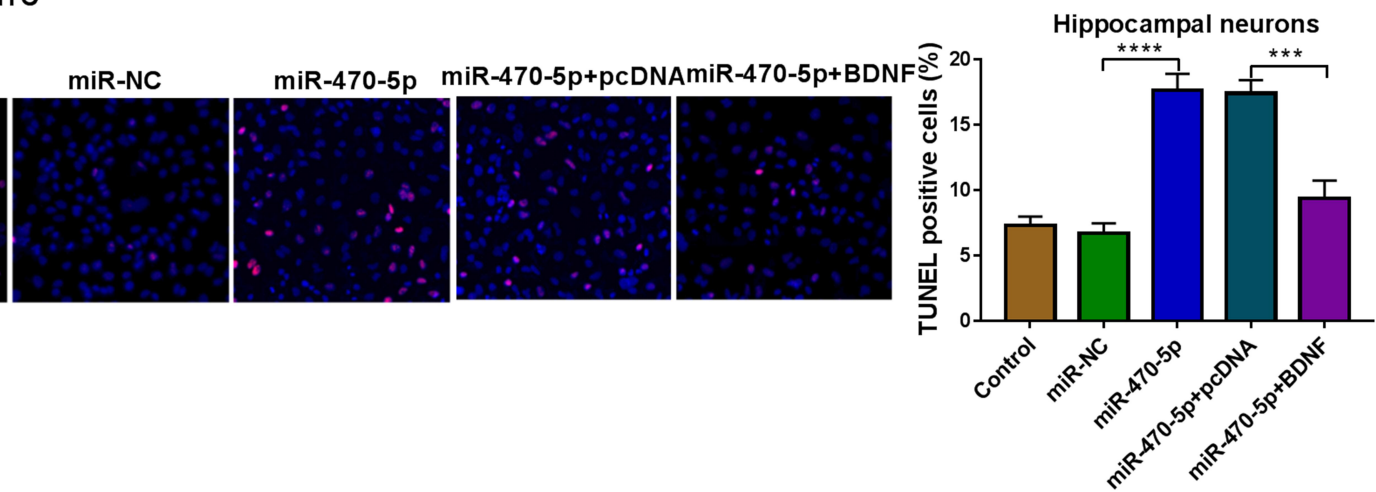

Figure 6 MiR-470-5p and BDNF regulated CUMS mice depressive behavior and hippocampal neurons growth. (A-D) C57BL/6] mice were performed CUMS program, and then injected with Ad-miR-NC, Ad-miR-470-5p, Ad-miR-470-5p + Ad-NC or Ad-miR-470-5p + Ad-BDNF. Non-treated mice were used as control. (A) BDNF expression was determined using WB analysis. SPT (B) and FST (C and D) were performed to examine the sucrose preference, swimming time and immobility time of mice. (E-H) Hippocampal neurons were transfected with miR-NC, miR-470-5p, miR-470-5p + pcDNA or miR-470-5p + BDNF. Non-transfected and non-treated cells were used as control. (E) The viability of cells was assessed using CCK8 assay. Cell apoptosis rate and TUNEL positive cells were determined by flow cytometry (F and $\mathbf{G})$ and TUNEL assay $(\mathbf{H})$ to evaluate cell apoptosis. $* P<0.05, * * P<0.01$, $* * * P<0.001$, $* * * * P<0.0001$. 


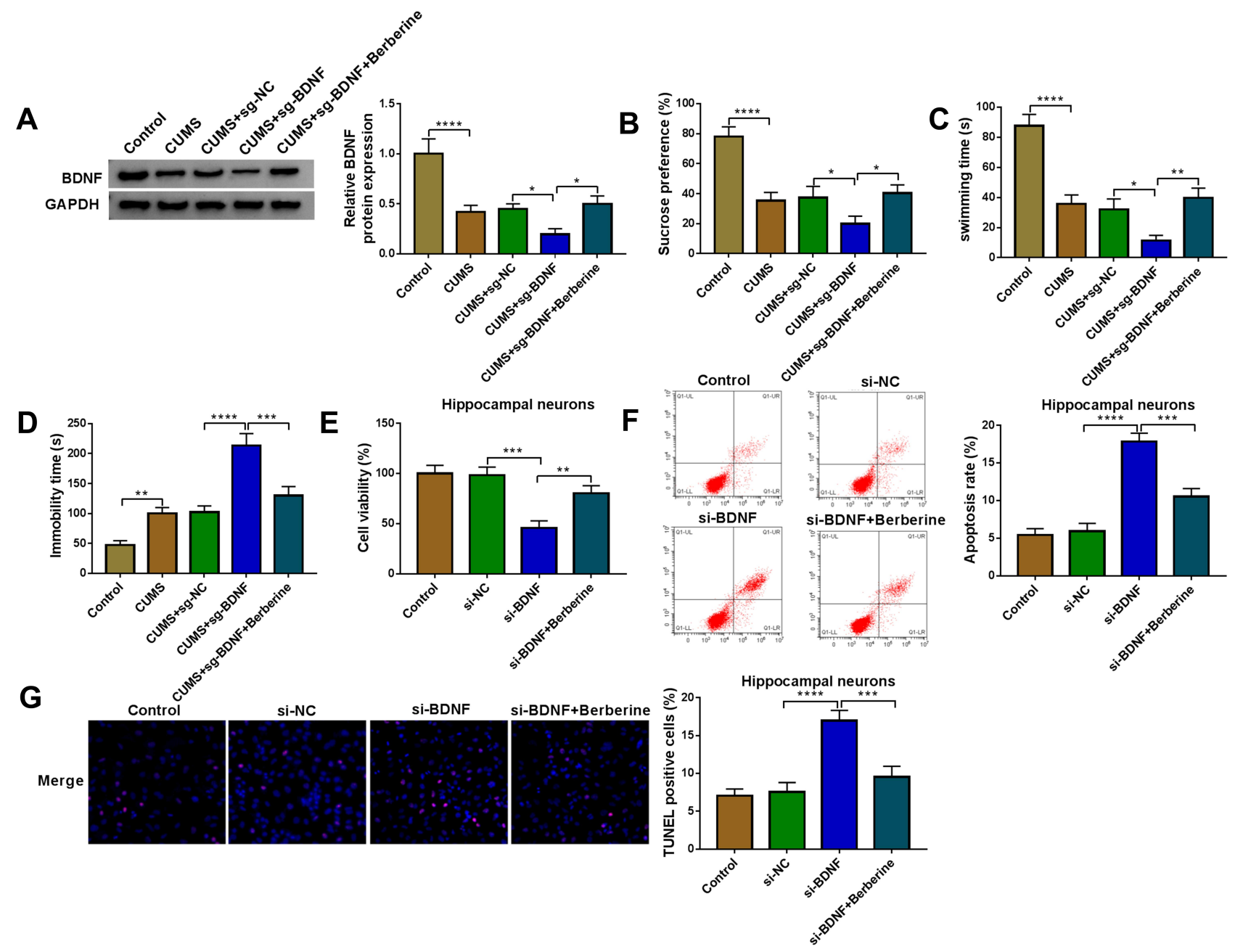

Figure 7 Berberine mediated BDNF expression to regulate CUMS mice depressive behavior and hippocampal neurons growth. (A-D) C57BL/6) mice were divided into 5 groups, including control, CUMS, CUMS + sg-NC, CUMS + sg-BDNF, CUMS + sg-BDNF + Berberine. (A) BDNF protein expression was determined using WB analysis. SPT (B) and FST (C-D) were used to evaluate the sucrose preference, swimming time and immobility time of mice. (E-G) Hippocampal neurons were transfected with si-NC or si-BDNF, or treated with Berberine. Non-transfected and non-treated cells were used as control. (E) The viability of cells was tested using CCK8 assay. Flow cytometry (F) and TUNEL assay $(\mathbf{G})$ were employed to detect cell apoptosis rate and TUNEL positive cells to assess cell apoptosis. $* P<0.05, * * P<0.01$, $* * * P<0.00 \mathrm{I}$, $* * * * P<0.000 \mathrm{I}$.

hippocampal neurons growth by regulating the miR-34b5p/miR-470-5p/BDNF axis (Figure 9).

\section{Discussion}

At present, Berberine has shown a significant antidepressant effect in the treatment of depression disorder, but its specific mechanism remains to be elucidated. There is evidence that a large number of miRNAs are specifically expressed or enriched in the brain or central nervous system, and the neurological disorders in depression patients are often accompanied by disorders in the expression of many miRNAs. ${ }^{33,34}$ MiRNAs have been proven to be used for the diagnosis and treatment biomarkers of depression. ${ }^{35,36}$ Here, we found that Berberine could regulate miR-34b-5p, miR-470-5p and BDNF expression, so we speculated that Berberine regulated the progression of depression disorder through miRNA/mRNA axis. Around this hypothesis, a series of experiments were carried out.

MiR-34b-5p has been found to be involved in the progression of many diseases. Li et al suggested that miR-34b$5 p$ overexpression could inhibit the oxidative stress injury of Parkinson's disease. ${ }^{37}$ And Dong et al reported that miR$34 \mathrm{~b}-5 \mathrm{p}$ could hinder breast cancer cell proliferation and metastasis. ${ }^{38}$ In the past reports, miR-470 was found to be associated with mouse embryonic stem cell differentiation, and its upregulation could promote cell transcriptional and morphological changes. ${ }^{39}$ Additionally, Li et al indicated that miR-470 could enhance the neoplastic transformation of liver cancer stem cells. ${ }^{40}$ However, there are few studies on the role of miR-34b-5p and miR-470-5p in depression 
A

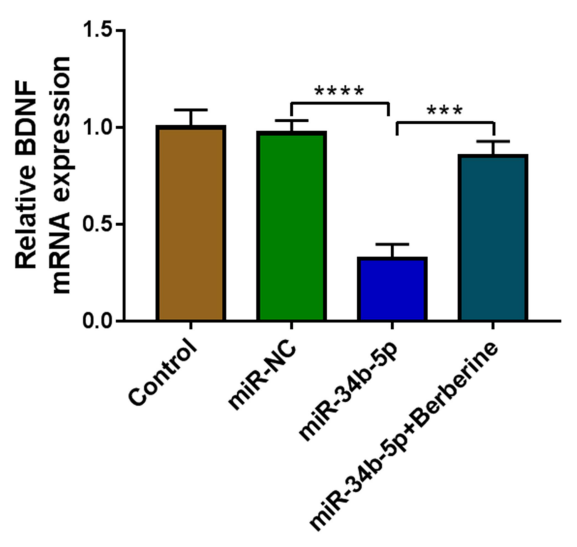

B

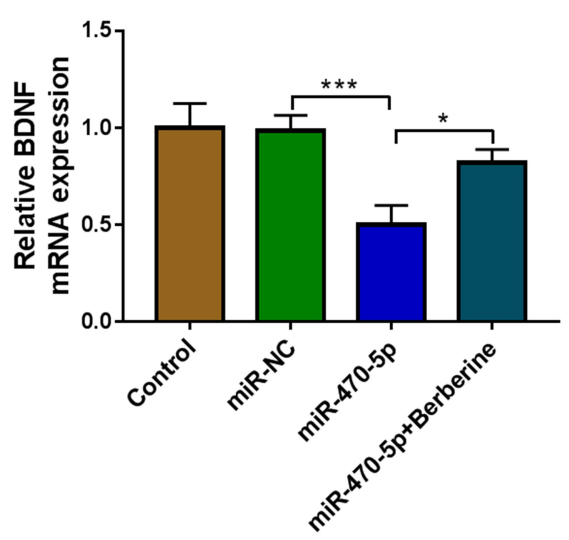

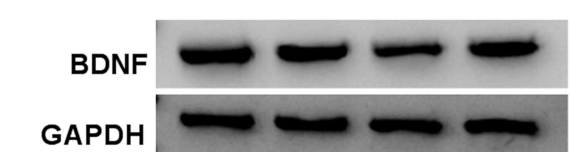
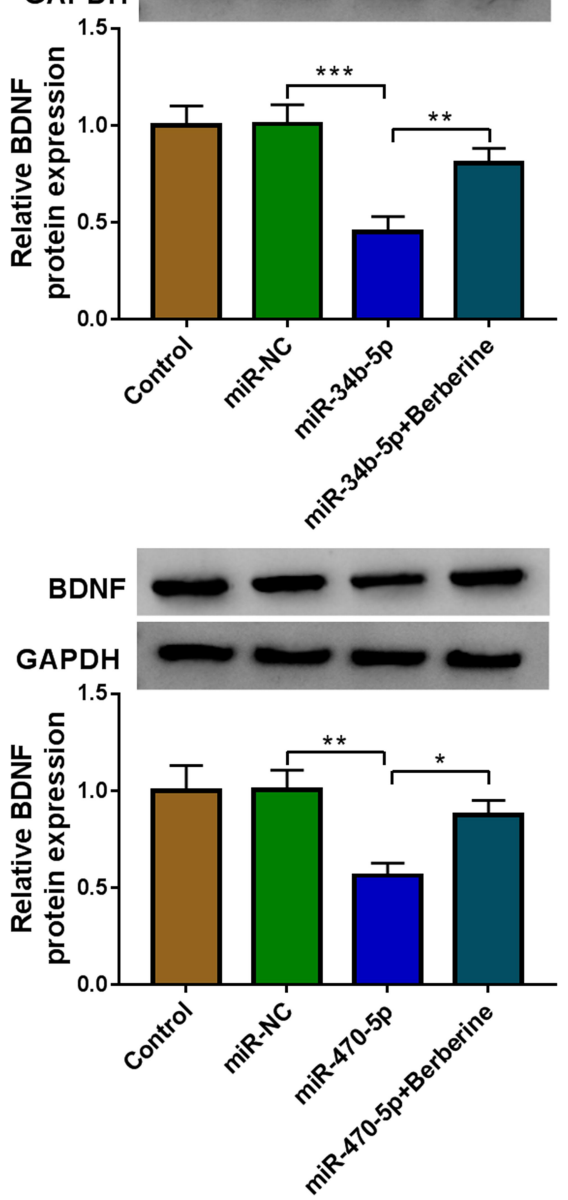

Figure 8 Berberine regulated BDNF expression by targeting miR-34b-5p and miR-470-5p in hippocampal neurons. Hippocampal neurons were transfected with miR-NC, miR-34b-5p or miR-470-5p, or treated with Berberine. Non-transfected and non-treated cells were used as control. (A and B) The mRNA and protein expression levels of BDNF were determined using $\mathrm{qRT}-\mathrm{PCR}$ and WB analysis. $* \mathrm{P}<0.05, * * P<0.01$, $* * * P<0.001, * * * * P<0.0001$.

disorder. Our study revealed that miR-34b-5p and miR-470$5 \mathrm{p}$ were markedly upregulated in the hippocampus of CUMS mice, which was consistent with the results of previous studies. $^{24,25}$ Further experiments revealed that Berberine could inhibit the expression levels of miR-34b$5 p$ and miR-470-5p in CUMS mice, and could reverse the promotion effect of miR-34b-5p and miR-470-5p on CUMS mice depressive behavior and the inhibition effect on hippocampal neurons growth. This evidence suggested that Berberine restrained the progression of depression disorder by regulating the expression of miR-34b-5p and miR-470$5 \mathrm{p}$, which was an important finding.

Many studies have indicated that BDNF expression is associated with the severity of illness in depressed patients, including suicidal ideation ${ }^{41}$ and executive dysfunctions. ${ }^{42}$ Therefore, BDNF has become a key biomarker for depression disorder. In our study, we uncovered that both miR-34b-5p and miR-470-5p could target BDNF. Moreover, BDNF also could reverse the increasing effect of miR-34b-5p and miR-470-5p on CUMS mice depressive behavior and the decreasing effect on hippocampal neurons growth. Importantly, we discovered that Berberine could promote BDNF expression in CUMS mice and could reverse the inhibition effect of miR-34b-5p and miR-470-5p on BDNF expression. Function experiments suggested that Berberine regulated the progression of depression disorder by mediating BDNF expression. These results revealed that Berberine was involved in the regulation of depression disorder progression through regulating the $\mathrm{miR}-34 \mathrm{~b}-5 \mathrm{p} / \mathrm{miR}$ 470-5p/BDNF axis, which was consistent with our speculation. 


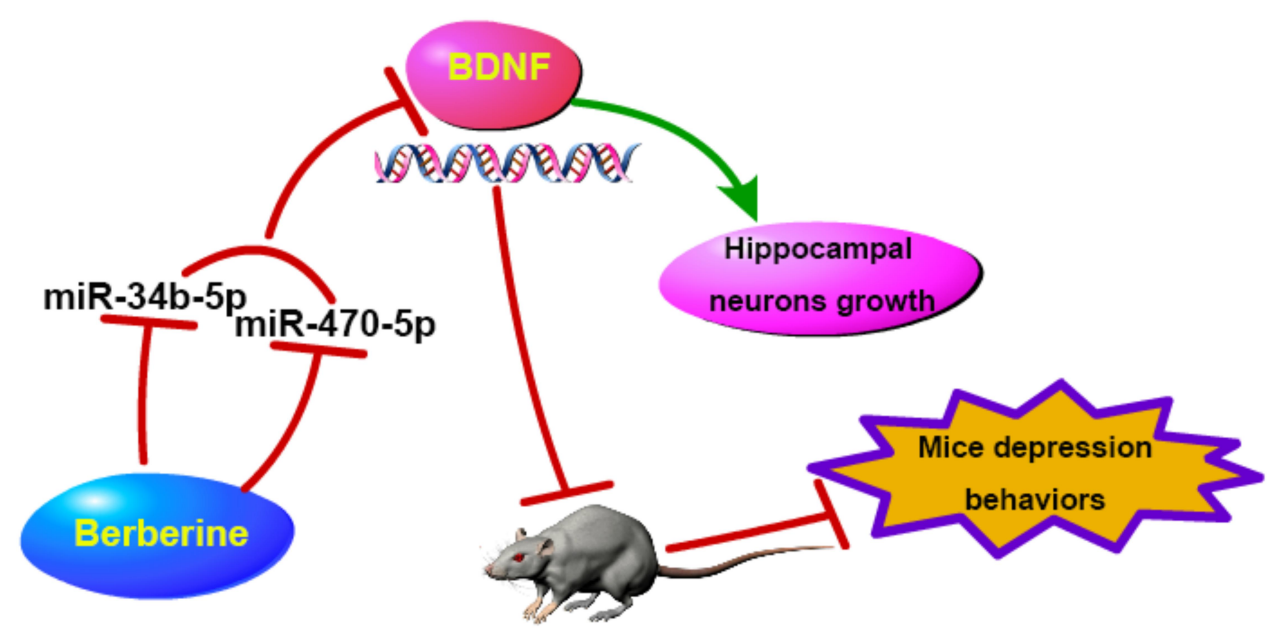

Figure 9 The schematic diagram of this study. Berberine inhibited CUMS mice depressive behavior and promoted hippocampal neurons growth, which was mainly achieved by regulating the miR-34b-5p/miR-470-5p/BDNF axis.

In summary, our data indicated that Berberine suppressed CUMS mice depression-like behaviors and promoted the growth of hippocampal neurons by targeting the miR-34b-5p/miR-470-5p/BDNF axis. The results of this study provided evidence for Berberine to inhibit the progression of depression disorder.

\section{Ethics Approval and Consent to Participate}

Our study was approved by the Ethics Committee of Shengjing Hospital of China Medical University.

\section{Funding}

This work was supported by Chengdu Municipal Health Commission Project (No.2019107).

\section{Disclosure}

The authors declare that they have no financial conflicts of interest.

\section{References}

1. Alexopoulos GS. Depression in the elderly. Lancet. 2005;365 (9475):1961-1970. doi:10.1016/S0140-6736(05)66665-2

2. Rakel RE. Depression. Prim Care. 1999;26(2):211-224. doi:10.1016/ S0095-4543(08)70003-4

3. Hauenstein EJ. Depression in adolescence. J Obstet Gynecol Neonatal Nurs. 2003;32(2):239-248. doi:10.1177/0884217503252133

4. Castiglia PT. Depression in children. J Pediatr Health Care. 2000;14 (2):73-75

5. Antoniuk S, Bijata M, Ponimaskin E, Wlodarczyk J. Chronic unpredictable mild stress for modeling depression in rodents: meta-analysis of model reliability. Neurosci Biobehav Rev. 2019;99:101-116.
6. Lu Q, Mouri A, Yang Y, et al. Chronic unpredictable mild stress-induced behavioral changes are coupled with dopaminergic hyperfunction and serotonergic hypofunction in mouse models of depression. Behav Brain Res. 2019;372:112053. doi:10.1016/j.bbr.2019.112053

7. Huang GB, Zhao T, Muna SS, et al. Effects of chronic social defeat stress on behaviour, endoplasmic reticulum proteins and choline acetyltransferase in adolescent mice. Int $J$ Neuropsychopharmacol. 2013;16(7):1635-1647. doi:10.1017/S1461145713000060

8. Zhao T, Huang GB, Muna SS, et al. Effects of chronic social defeat stress on behavior and choline acetyltransferase, $78-\mathrm{kDa}$ glucose-regulated protein, and CCAAT/enhancer-binding protein $(\mathrm{C} /$ EBP) homologous protein in adult mice. Psychopharmacology. 2013;228(2):217-230. doi:10.1007/s00213-013-3028-6

9. Bagalkot TR, Jin HM, Prabhu VV, et al. Chronic social defeat stress increases dopamine D2 receptor dimerization in the prefrontal cortex of adult mice. Neuroscience. 2015;311:444-452. doi:10.1016/j. neuroscience.2015.10.024

10. Jin HM, Shrestha Muna S, Bagalkot TR, Cui Y, Yadav BK, Chung YC. The effects of social defeat on behavior and dopaminergic markers in mice. Neuroscience. 2015;288:167-177. doi:10.1016/j. neuroscience.2014.12.043

11. Nollet M, Le Guisquet AM, Belzung C. Models of depression: unpredictable chronic mild stress in mice. Curr Protoc Pharmacol. 2013; Chapter 5(Unit 5):65. doi:10.1002/0471141755.ph0565s61

12. Koprdova R, Bogi E, Belovicova K, et al. Chronic unpredictable mild stress paradigm in male Wistar rats: effect on anxiety- and depressive-like behavior. Neuro Endocrinol Lett. 2016;37 (Suppl1):103-110.

13. Li C, Xie J, Chen X, et al. Comparison of Helicobacter pylori urease inhibition by rhizoma coptidis, cortex phellodendri and Berberine: mechanisms of interaction with the sulfhydryl group. Planta Med. 2016;82(4):305-311. doi:10.1055/s-0035-1558229

14. Fujii A, Okuyama T, Wakame K, Okumura T, Ikeya Y, Nishizawa M. Identification of anti-inflammatory constituents in Phellodendri Cortex and Coptidis Rhizoma by monitoring the suppression of nitric oxide production. J Nat Med. 2017;71(4):745-756. doi:10.1007/ s11418-017-1107-4

15. Cicero AF, Baggioni A. Berberine and its role in chronic disease. $A d v$ Exp Med Biol. 2016;928:27-45.

16. Kulkarni SK, Dhir A. Berberine: a plant alkaloid with therapeutic potential for central nervous system disorders. Phytother Res. 2010;24(3):317-324. doi:10.1002/ptr.2968 
17. Zhu X, Sun Y, Zhang C, Liu H. Effects of berberine on a rat model of chronic stress and depression via gastrointestinal tract pathology and gastrointestinal flora profile assays. Mol Med Rep. 2017;15 (5):3161-3171. doi:10.3892/mmr.2017.6353

18. Fan J, Li B, Ge T, et al. Berberine produces antidepressant-like effects in ovariectomized mice. Sci Rep. 2017;7(1):1310. doi:10.1038/s41598-017-01035-5

19. Liu YM, Niu L, Wang LL, et al. Berberine attenuates depressive-like behaviors by suppressing neuro-inflammation in stressed mice. Brain Res Bull. 2017;134:220-227. doi:10.1016/j.brainresbull.2017.08.008

20. Hu Z, Jiang Y, Huo X, et al. Prospective role of microRNAs in depression. Curr Med Chem. 2017;24(32):3508-3521. doi:10.2174/ 0929867324666170714112620

21. Tavakolizadeh J, Roshanaei K, Salmaninejad A, et al. MicroRNAs and exosomes in depression: potential diagnostic biomarkers. J Cell Biochem. 2018;119(5):3783-3797. doi:10.1002/jcb.26599

22. Hung YY, Wu MK, Tsai MC, Huang YL, Kang HY. Aberrant expression of intracellular let-7e, miR-146a, and miR-155 correlates with severity of depression in patients with major depressive disorder and is ameliorated after antidepressant treatment. Cells. 2019;8(7):7. doi: $10.3390 /$ cells 8070647

23. Sun N, Lei L, Wang Y, et al. Preliminary comparison of plasma notch-associated microRNA-34b and $-34 \mathrm{c}$ levels in drug naive, first episode depressed patients and healthy controls. J Affect Disord. 2016;194:109-114. doi:10.1016/j.jad.2016.01.017

24. Ma K, Zhang H, Wei G, et al. Identification of key genes, pathways, and miRNA/mRNA regulatory networks of CUMS-induced depression in nucleus accumbens by integrated bioinformatics analysis. Neuropsychiatr Dis Treat. 2019;15:685-700. doi:10.2147/NDT. S200264

25. Ma K, Guo L, Xu A, Cui S, Wang JH. Molecular mechanism for stress-induced depression assessed by sequencing miRNA and mRNA in medial prefrontal cortex. PLoS One. 2016;11(7): e0159093. doi:10.1371/journal.pone.0159093

26. Numakawa T, Suzuki S, Kumamaru E, Adachi N, Richards M, Kunugi H. BDNF function and intracellular signaling in neurons. Histol Histopathol. 2010;25(2):237-258. doi:10.14670/HH-25.237

27. von Bohlen Und Halbach O, von Bohlen Und Halbach V. BDNF effects on dendritic spine morphology and hippocampal function. Cell Tissue Res. 2018;373(3):729-741. doi:10.1007/s00441-0172782-x

28. Bjorkholm C, Monteggia LM. BDNF - a key transducer of antidepressant effects. Neuropharmacology. 2016;102:72-79. doi:10.1016/ j.neuropharm.2015.10.034

29. Mondal AC, Fatima M. Direct and indirect evidences of BDNF and NGF as key modulators in depression: role of antidepressants treatment. Int J Neurosci. 2019;129(3):283-296. doi:10.1080/ 00207454.2018 .1527328
30. Li C, Wang F, Miao P, et al. miR-138 increases depressive-like behaviors by targeting SIRT1 in hippocampus. Neuropsychiatr Dis Treat. 2020;16:949-957. doi:10.2147/NDT.S237558

31. Lian N, Niu Q, Lei Y, Li X, Li Y, Song X. MiR-221 is involved in depression by regulating Wnt2/CREB/BDNF axis in hippocampal neurons. Cell Cycle. 2018;17(24):2745-2755. doi:10.1080/ 15384101.2018.1556060

32. Wang Y, Xu J, Liu Y, Li Z, Li X. TLR4-NF-kappaB signal involved in depressive-like behaviors and cytokine expression of frontal cortex and hippocampus in stressed C57BL/6 and ob/ob mice. Neural Plast. 2018;2018:7254016. doi:10.1155/2018/7254016

33. Ferrua CP, Giorgi R, da Rosa LC, et al. MicroRNAs expressed in depression and their associated pathways: a systematic review and a bioinformatics analysis. J Chem Neuroanat. 2019;100:101650. doi:10.1016/j.jchemneu.2019.101650

34. Fries GR, Zhang W, Benevenuto D, Quevedo J. MicroRNAs in major depressive disorder. Adv Exp Med Biol. 2019;1118:175-190.

35. Lopez JP, Kos A, Turecki G. Major depression and its treatment: microRNAs as peripheral biomarkers of diagnosis and treatment response. Curr Opin Psychiatry. 2018;31(1):7-16. doi:10.1097/ YCO.0000000000000379

36. Xu YY, Xia QH, Xia QR, Zhang XL, Liang J. MicroRNA-based biomarkers in the diagnosis and monitoring of therapeutic response in patients with depression. Neuropsychiatr Dis Treat. 2019;15:3583-3597. doi:10.2147/NDT.S237116

37. Li Y, Fang J, Zhou Z, et al. Downregulation of lncRNA BACE1-AS improves dopamine-dependent oxidative stress in rats with Parkinson's disease by upregulating microRNA-34b-5p and downregulating BACE1. Cell Cycle. 2020;19(10):1158-1171. doi:10.1080/ 15384101.2020.1749447

38. Dong L, Chen F, Fan Y, Long J. MiR-34b-5p inhibits cell proliferation, migration and invasion through targeting ARHGAP1 in breast cancer. Am J Transl Res. 2020;12(1):269-280.

39. Tay Y, Zhang J, Thomson AM, Lim B, Rigoutsos I. MicroRNAs to Nanog, Oct4 and Sox2 coding regions modulate embryonic stem cell differentiation. Nature. 2008;455(7216):1124-1128. doi:10.1038/ nature07299

40. Li R, Qian N, Tao K, You N, Wang X, Dou K. MicroRNAs involved in neoplastic transformation of liver cancer stem cells. J Exp Clin Cancer Res. 2010;29(1):169. doi:10.1186/1756-9966-29-169

41. Khan MS, Wu GWY, Reus VI, et al. Low serum brain-derived neurotrophic factor is associated with suicidal ideation in major depressive disorder. Psychiatry Res. 2019;273:108-113. doi:10.1016/j.psychres.2019.01.013

42. Wagner S, Kayser S, Engelmann J, et al. Plasma brain-derived neurotrophic factor (pBDNF) and executive dysfunctions in patients with major depressive disorder. World J Biol Psychiatry. 2019;20 (7):519-530. doi:10.1080/15622975.2018.1425478
Neuropsychiatric Disease and Treatment

\section{Publish your work in this journal}

Neuropsychiatric Disease and Treatment is an international, peerreviewed journal of clinical therapeutics and pharmacology focusing on concise rapid reporting of clinical or pre-clinical studies on a range of neuropsychiatric and neurological disorders. This journal is indexed on PubMed Central, the 'PsycINFO' database and CAS, and is the official journal of The International Neuropsychiatric Association (INA). The manuscript management system is completely online and includes a very quick and fair peer-review system, which is all easy to use. Visit http://www.dovepress.com/testimonials.php to read real quotes from published authors. 